\title{
Strates
}

STRATES Matériaux pour la recherche en sciences sociales

15 | 2008

Union européenne - Voisinages. La quête d'une intégration régionale

\section{Eau et assainissement des villes et pays riverains de la Méditerranée}

Claude Martinand et Marie-Joëlle Kodjovi

\section{(2) OpenEdition}

\section{Journals}

Édition électronique

URL : http://journals.openedition.org/strates/6546

DOI : $10.4000 /$ strates.6546

ISSN : $1777-5442$

Éditeur

Laboratoire Ladyss

Édition imprimée

Date de publication : 1 janvier 2008

Pagination : 129-142

ISSN : 0768-8067

Référence électronique

Claude Martinand et Marie-Joëlle Kodjovi, «Eau et assainissement des villes et pays riverains de la Méditerranée », Strates [En ligne], 15 | 2008, mis en ligne le 22 janvier 2013, consulté le 08 septembre 2020. URL : http://journals.openedition.org/strates/6546 ; DOI : https://doi.org/10.4000/strates.6546

Ce document a été généré automatiquement le 8 septembre 2020

Tous droits réservés 


\title{
Eau et assainissement des villes et pays riverains de la Méditerranée
}

\author{
Claude Martinand et Marie-Joëlle Kodjovi
}

1 La Méditerranée et ses rives constituent un patrimoine naturel et historique exceptionnel dont la préservation est depuis plus de trente ans un objectif partagé par les pays riverains et le Programme des Nations unies pour l'environnement (PNUE). Notre «mer commune » quasi fermée reçoit, accumule et concentre l'ensemble des pollutions émises par les villes et pays riverains ainsi que par les activités maritimes elles-mêmes. Dès lors que $80 \%$ de ces pollutions sont d'origine terrestre, s'intéresser à l'assainissement des villes, notamment côtières, paraît une approche prioritaire d'autant plus que les activités et les populations ont tendance à se concentrer sur la bande littorale et que les éléments de prospective confirment cette tendance pour les décennies à venir.

2 Il ne s'agit pas de reprendre ce que de multiples conventions, plans et programmes ou fonds d'investissements ont tenté de mettre en œuvre depuis la Convention de Barcelone de 1975 et le Plan d'action pour la Méditerranée (PAM) élaborés sous l'égide des Nations unies, de l'Union européenne et de l'ensemble des pays concernés. L'historique des dispositions adoptées en commun et des actions engagées pour réduire les pollutions montre l'ampleur du dispositif mis en place et l'élargissement progressif des préoccupations depuis la préservation et la mise en valeur de l'environnement jusqu'à une véritable stratégie méditerranéenne de développement durable: élaboration d'Agendas 21 décidée à Tunis en 1994, révision du PAM à Barcelone en 1995 et création d'une Commission méditerranéenne du développement durable (CMDD) décidée à Montpellier en 1996. Ce dispositif est complété par d'autres actions lancées par l'Union européenne (L'instrument financier pour l'environnement - Life, le programme d'actions à court et moyen terme - le Smap, l'initiative « Horizon 2020 »), ou dans le cadre des Objectifs du millénaire pour le développement adoptés par l'ONU en 2000 , dont la lutte contre la pauvreté et l'accès à l'eau potable et à l'assainissement font partie. 
3 Mais tant au niveau méditerranéen qu'au niveau mondial, le rendez-vous de l'ONU en 2005 («OMD+5») a permis de constater l'insuffisance des résultats obtenus et l'impossibilité d'atteindre, à ce rythme, les objectifs adoptés, notamment en Afrique. Il est vrai qu'au fur et à mesure que des résultats sont atteints, l'urbanisation côtière rapide génère de nouveaux besoins, à un rythme plus rapide encore que celui constaté pour les résoudre. Les villes qui sont des « aimants de l'espoir » et attirent vers elles de nouveaux migrants ruraux, manquent souvent des outils pour organiser leur développement et réaliser, en temps réel, les équipements urbains souhaitables. Cependant, il faut reconnaître que certains pays ont engagé de longue date des politiques sérieuses et ambitieuses qui ont montré leur efficacité comme la Tunisie.

Eau et assainissement en Tunisie, éléments d'un projet politique : l'émergence d'une classe moyenne

Dès l'indépendance, la desserte en eau et assainissement est apparue comme un élément contribuant à l'émergence d'une classe moyenne à travers un habitat décent. Les solutions retenues ont conduit à la mise en place d'établissements publics nationaux (Sonde et Onas), qui ont progressivement étendu leurs actions à l'ensemble du pays. Le pays, de taille limité il est vrai, a su ainsi trouver des solutions efficaces. Progressivement, une partie des missions est désormais déléguée à des opérateurs privés.

D'autres pays se sont engagés plus récemment dans la recherche de solutions novatrices, le Maroc et l'Algérie par exemple. Faut-il rappeler que les villes de Marseille, Nice ou Toulon ne se sont dotées de politiques de lutte contre la pollution marine que dans un passé fort récent? Il s'agit donc de s'interroger sur les raisons qui ont conduit à des résultats aussi mitigés, certes variables selon les pays, mais dans l'ensemble insuffisants.

5 Tout d'abord, il est apparu que traiter la question prioritaire de l'assainissement des villes impliquait de la replacer dans le cadre d'une gestion globaledu service d'eau et d'assainissement, de la production et distribution de l'eau potable à la collecte et au traitement des eaux usées, s'intégrant dans le cycle naturel de la ressource et permettant un équilibre entre ses multiples usages (irrigation, utilisations industrielles, consommation des ménages). La nécessaire gestion intégrée du cycle de l'eau dans des territoires pertinents (bassins fluviaux généralement), abordée dans d'autres cadres, ne fait pas l'objet du présent travail. Plus généralement, il est apparu qu'aborder la question de l'eau et de l'assainissement sans la replacer dans une approche intégrant différentes questions (l'aménagement des territoires, le développement économique, les progrès de la société en particulier la lutte contre la pauvreté ou la santé des populations, les préoccupations de solidarité et d'équité, la bonne gouvernance ou la lutte contre la corruption), constitue un obstacle pour aborder efficacement la lutte contre les pollutions.

Le problème doit donc être abordé à travers une véritable démarche de développement durable reposant sur ses trois piliers : le développement économique, le progrès de la société, la protection et la mise en valeur de l'environnement. Le développement durable ne saurait être confondu avec son volet environnemental. Il relève d'une approche transversale, intégrée, impliquant de multiples politiques publiques abordées trop souvent de manière sectorielle sans coordination suffisante. Une politique de 
développement durable implique de bons diagnostics et des visions partagées entre les différents acteurs concernés. Cela suppose d'élaborer un langage commun pour éviter des contresens ou des malentendus.

$7 \quad$ En définitive, les études de cas montrent que c'est bien l'insuffisante mise en œuvre des principes du développement durable qui constitue la difficulté centrale des problèmes à résoudre. L'assainissement des eaux urbaines et industrielles ne constitue que rarement une priorité politique placée en haut de l'Agenda. Ce problème ne peut être résolu par les seuls ministres de l'environnement. Les approches économiques, sociales et sanitaires relevant d'autres ministères sont des entrées pertinentes pour s'attaquer ensuite à la desserte en eau et surtout à l'assainissement. Identifier les objectifs les plus mobilisateurs et les acteurs clefs à mobiliser sur ces objectifs partagés constitue donc un préalable.

8 Le groupe de travail n'a pas considéré que les problèmes de financement ou du choix du mode de gestion constituaient les questions centrales. D'une part, il a montré le rôle crucial de la politique tarifaire pour garantir la pérennité du service, l'équité et la solidarité. D'autre part, il a identifié trois questions clefs pour réussir : l'amélioration de la gouvernance et la maîtrise publique du service; le développement des compétences des autorités responsables et des opérateurs (capacité à réaliser et à gérer); l'amélioration continue de la performance du service (qualité et efficacité économique). Quant au mode de gestion, il faut sortir des débats idéologiques, constater la pluralité des solutions et privilégier les relations contractuelles assorties de mécanismes incitatifs entre l'autorité publique et l'opérateur, qu'il soit public ou privé.

\section{Le diagnostic du problème}

\section{Quels sont les objectifs mobilisateurs?}

9 Même s'il faut le regretter, les objectifs de lutte contre les pollutions ne sont que rarement considérés comme prioritaires dans les pays en développement. En outre, les ministres de l'environnement, en charge de la négociation de ces grandes conventions, ne sont pas toujours les mieux placés pour les mettre en œuvre car d'autres ministères (travaux publics, hydraulique, intérieur) sont plus directement en charge des équipements et des services urbains auprès des autorités locales. Le relais n'est souvent pas pris entre ces différents ministères, préoccupés par de multiples autres priorités auxquelles ils doivent faire face avec des moyens limités. La lutte contre la pauvreté, la santé publique et la politique de l'habitat (un habitat décent pour les plus démunis) relèvent également d'autres autorités. Cette dimension sociale est sans doute mieux perçue, mais les solutions à y apporter ne sont pas faciles non plus à imaginer et à mettre en œuvre. Le développement économique est sans doute l'entrée la plus mobilisatrice pour les gouvernants, car elles entraînent des créations d'emplois, de revenus et de ressources, y compris fiscales.

10 En fait, les solutions à ces différents problèmes sont liées et c'est en les abordant simultanément et de manière intégrée que l'on a des chances de progresser. Par exemple, le tourisme exige des plages et des eaux marines propres pour attirer les touropérateurs et donc des investissements d'accueil mais aussi des stations d'épuration efficaces. Le tourisme est manifestement une source importante de développement 
économique durable, de devises et d'emplois dans nombre de pays concernés. Là encore, cela implique de mobiliser l'ensemble des ministères concernés et notamment ceux de l'Intérieur, de l'Économie et des Finances. Alors les démarches de développement durable peuvent se concrétiser et dépasser le stade des déclarations d'intention.

\section{Quels acteurs mobiliser sur ces objectifs partagés?} un processus de stabilisation et de consolidation que si la question foncière est « régularisée » et les dessertes en eau, en assainissement liquide et solide et en électricité sont mises en place en parallèle. Alors, un processus vertueux de création d'emplois (artisans, petits ateliers industriels), de richesses, et d'émergence d'une véritable classe moyenne peut s'engager. La Tunisie dès l'indépendance, et le Maroc plus récemment avec l'Initiative nationale pour le développement humain (INDH) montrent l'efficacité de ces démarches globales liant les trois piliers du développement durable.

Eau et assainissement, composantes de politiques intégrées. Deux exemples marocains

La composante eau et assainissement de l'INDH

Dans le cadre de l'Initiative nationale de développement humain, un programme de desserte en eau potable et en assainissement des quartiers les plus pauvres a été lancé au Maroc. Il contribue à l'atteinte des objectifs de la politique nationale d'amélioration des conditions de vie des populations logeant dans l'habitat insalubre, souvent informel, selon un plan d'aménagement global des 
agglomérations avec maintien des populations sur place et régularisation foncière. Il s'agit ainsi de susciter un développement local engendrant emplois, ressources, revenus et amélioration de l'habitat. À Casablanca, la société Lydec est chargée de l'ingénierie sociale (50 personnes) nécessaire à la desserte en eau, en assainissement et en électricité de 137000 foyers résidant dans l'habitat insalubre informel. Dès 2006,16000 foyers, soit 47000 personnes, ont déjà été pris en charges par des opérations qui ont démarré.

L'eau et l'assainissement, éléments de l'aménagement de la région Tanger - Tétouan

L'avènement du nouveau roi Mohammed VI a mis l'accent sur le développement économique du Nord du Maroc, au plan industriel et touristique. Dans ce cadre, la maîtrise de la distribution de l'eau potable et de l'assainissement des eaux usées pour éviter des rejets polluants en mer, est apparue prioritaire. Des conventions de gestion déléguée ont été conclues, après mise en compétition, avec une société franco marocaine, Amendis, opérant dans le cadre de relations équilibrées public (autorités publiques) - privé (société délégataire). Le programme INDH bénéficiant de multiples fonds publics et dons, permet simultanément de faire accéder les ménages les plus modestes à l'eau potable et à l'assainissement.

Les opérateurs de service en place ou susceptibles d'intervenir, publics ou privés, doivent être consultés et impliqués dans les diagnostics et les démarches de progrès envisagées.

\section{Trois questions clefs pour réussir}

En matière d'approche intégrée du développement durable, trois questions prioritaires devraient faire simultanément l'objet de réponses satisfaisantes :

\section{L'amélioration de la gouvernance et de la maîtrise publique du service}

17 Il s'agit de clarifier qui est responsable de quoi, quelles sont les missions à accomplir par les uns ou par les autres et quelles sont les relations, de préférence contractuelles, à établir entre les principaux acteurs.

18 Le niveau national doit fixer le cadre réglementaire, les objectifs généraux à atteindre, les normes à respecter, et mettre en place les moyens, définir les contrôles à effectuer et évaluer les résultats, bref se doter d'une véritable «régulation». Si l'État peut conserver l'organisation directe du service, au moins dans une première phase, il doit, on l'a dit, impliquer les autorités locales.

Les opérateurs doivent être responsables de l'exploitation des services selon des objectifs de niveau de service et des règles fixées par les autorités publiques. Ils doivent être clairement placés sous le contrôle de leurs résultats par les autorités publiques. Il faut absolument éviter la confusion et l'inversion des rôles entre les opérateurs et les autorités publiques, les opérateurs fixant les objectifs et les autorités publiques s'ingérant dans la gestion quotidienne. Les salariés des opérateurs peuvent eux-mêmes être consultés et intéressés aux résultats. 
20 Les bénéficiaires $d u$ service doivent être impliqués sur les questions où ils peuvent jouer un rôle positif, dès lors qu'ils sont convenablement informés. Il peut paraître judicieux d'assurer leur représentation en distinguant les différentes catégories d'usagers domestiques, agricoles et industriels.

21 Les périmètres pertinents de cette gouvernance doivent être déterminés en fonction des réalités techniques, géographiques et humaines, d'autant plus que cela conditionnera les politiques futures de péréquations. Les relations entre les acteurs doivent avoir pour objectif de construire la confiance et le partenariat. Les solutions contractuelles en sont la forme la plus évoluée car elles placent les partenaires sur un pied d'égalité et favorisent les jeux gagnant-gagnant à travers des mécanismes incitatifs.

\section{Le développement des compétences des autorités responsables et des opérateurs (capacité à réaliser et à gérer)}

22 Les ressources humaines sont cruciales et doivent être mobilisées aux niveaux où elles peuvent concentrer une masse critique suffisante. Les compétences nécessaires sont de nature autant technique, économique, juridique, administrative et financière que managériale ou commerciale. Elles doivent être différenciées selon qu'elles concernent :

23 - l'élaboration d'une politique, d'un diagnostic, la fixation des objectifs à atteindre, l'évaluation des résultats, la capacité à définir des programmes d'investissements et à étudier des projets, à organiser la mise en compétition, à négocier avec l'exploitant et à le contrôler;

24 - la capacité à exploiter le service de manière aussi professionnelle que possible et à gérer les ressources humaines, les process industriels et les procédures.

25 À défaut d'une compétence interne suffisante, il faut la renforcer à travers des plans de formation et en recourant à des compétences externes que l'on peut trouver chez des consultants, des experts ou des professionnels reconnus, ou auprès des ONG et des organisations internationales. La coopération décentralisée et les échanges entre villes du Nord et du Sud sont des voies intéressantes, dès lors qu'elles s'inscrivent dans la durée et recouvrent des sujets de fond abordés sur des bases claires. La coopération technique ou l'ingénierie sociale sont des exemples.

\section{L'engagement d'un processus d'amélioration de la performance globale du service (qualité et productivité)}


le réseau. En ce qui concerne l'assainissement, le taux de raccordement au réseau de collecte des eaux usées, le taux de traitement de ces eaux usées (efficacité de l'épuration) et le volume des rejets directs en mer, même avec des émissaires, permettent d'apprécier l'efficacité de la politique mise en œuvre. Enfin pour ces deux services, le taux de réclamation, le prix en fonction du niveau de vie des ménages et le taux d'impayés sur les factures d'eau sont des indicateurs significatifs.

Un processus vertueux d'amélioration continue des résultats nécessite: un management responsable et aussi professionnel que possible, une bonne gestion des ressources humaines et une meilleure motivation des agents (formation, management participatif, intéressement aux résultats...), une bonne connaissance des usagers en développant une démarche de service (information, réponses aux réclamations, transparence et, même, démarche de marketing), des projets de service et des contrats d'objectifs comportant des objectifs de gains de productivité et de qualité conformément à des indicateurs de performance permettant la comparaison et l'émulation avec les services d'autres villes comparables (benchmarking).

Dès lors que des gains de productivité sont dégagés, il est nécessaire de prévoir une juste répartition du surplus économique. On peut imaginer qu'une première partie soit consacrée à l'autofinancement $\mathrm{du}$ branchement de nouveaux usagers et du renouvellement ou de l'extension ou de la modernisation des infrastructures; une seconde partie aux usagers, soit à travers des améliorations de qualité soit à travers des contributions aux péréquations tarifaires; et une troisième partie aux salariés qui ont permis ces gains de productivité et de qualité. Cette équité et plus généralement cette éthique soulèvent clairement la question d'une bonne régulation et d'un contrôle aussi démocratique que possible où toutes les parties prenantes, usagers et salariés compris, peuvent jouer un rôle utile.

\section{Une question centrale : une politique tarifaire conciliant pérennité du service, équité et solidarité}

\section{Éviter la fausse bonne idée de la gratuité et la sous tarification généralisée}

32 L'eau potable et l'assainissement urbain étant des services publics industriels et commerciaux, ils sont offerts en contrepartie d'un tarif d'ordre public fixé par l'autorité publique compétente, "l'autorité organisatrice». D'une manière ou d'une autre, la totalité des coûts doit être couverte par des tarifs et des contributions publiques pour assurer l'équilibre financier du service. La politique tarifaire constitue à la fois l'instrument de pilotage économique et financier du service, et l'outil de la solidarité entre les usagers et entre les territoires.

Certains invoquent l'incapacité des plus démunis à payer ce «bien public» pour préconiser la gratuité, nonobstant ses multiples effets pervers: le gaspillage d'une ressource rare, la dégradation du patrimoine non entretenu, la mauvaise gestion d'un service dont les coûts ne sont pas convenablement couverts et la nécessité permanente de subventions de fonctionnement. La réponse aux difficultés de paiement des plus démunis passe par la modulation tarifaire fondée sur des péréquations et par des aides ciblées, réservées à ceux qui en ont le plus besoin, sans effet d'aubaine pour les autres. 


\section{Mettre en place des mécanismes de solidarité par la modulation tarifaire et des aides ciblées}

être mises en place en faveur de ceux qui en ont impérieusement besoin : quantité plafonnée d'eau gratuite, subvention au tarif... Toutefois les populations non raccordées, en général les plus pauvres, sont exclues de ces mécanismes d'aide, et paient l'eau potable souvent fort cher aux porteurs d'eau ou aux gestionnaires de bornes-fontaines. Aussi, une subvention à la facture d'eau est d'autant plus pertinente que la plupart des usagers sont déjà bien raccordés. Sinon, il faut privilégier les subventions aux branchements pour l'accès à l'eau.

Le contrat global eau-assainissement-électricité de Casablanca (Lydec)

Le contrat de Casablanca, confié à Lydec en 1997, met en œuvre une péréquation originale entre les trois services publics de distribution de l'eau potable, de l'assainissement et de la distribution de l'électricité. La production d'eau et d'électricité est réalisée par ailleurs dans le cadre des Offices Publics compétents. Cet ensemble, qui a bien fonctionné dans d'autres pays d'Afrique (Côte d'Ivoire), permet de mieux couvrir les coûts d'exploitation à travers une péréquation entre les tarifs aidés de l'eau et de l'assainissement et ceux de l'électricité, dont les usagers professionnels sont prépondérants. En outre, la péréquation en faveur des populations défavorisées est assurée sur une agglomération de 4 millions d'habitants, où elle trouve son fondement dans les différences de capacité contributives selon les quartiers.

Des aides ciblées, évitant tout effet d'aubaine pour les usagers aisés, peuvent également être mises en place en faveur de ceux qui en ont impérieusement besoin : quantité plafonnée d'eau gratuite, subvention au tarif... Toutefois les populations non raccordées, en général les plus pauvres, sont exclues de ces mécanismes d'aide, et paient l'eau potable souvent fort cher aux porteurs d'eau ou aux gestionnaires de bornes-fontaines. Aussi, une subvention à la facture d'eau est d'autant plus pertinente 
que la plupart des usagers sont déjà bien raccordés. Sinon, il faut privilégier les subventions aux branchements pour l'accès à l'eau.

S'il faut donc éviter la fausse bonne idée de la gratuité ou de la sous tarification généralisée, il est réciproquement important d'accepter si nécessaire de tarifer l'eau en dessous du coût complet pour les usagers domestiques. L'histoire des pays développés montre que des crédits publics massifs ont été constamment nécessaires, au moins pour les équipements primaires des villes, pour l'adduction d'eau rurale et pour l'assainissement, et que les processus d'équipement se sont étalés sur de nombreuses décennies. L'assainissement urbain s'achève à peine en France dans le cadre des obligations communautaires. Comment imaginer que des pays en développement arrivent à réaliser en 20 ou 30 ans un effort d'équipement similaire par autofinancement des investissements à travers les résultats de l'exploitation des services? Par contre, la tarification doit couvrir au moins les coûts d'entretien et d'exploitation du service (" petit équilibre »), pour assurer la pérennité du service.

L'amélioration de la performance doit permettre d'accroître progressivement l'autofinancement pour couvrir tout ou partie des coûts de renouvellement du réseau, de manière à maintenir le patrimoine en bon état et assurer la durabilité du service. Les coûts de développement du réseau et notamment d'extension du réseau dans les quartiers spontanés ne peuvent être couverts par la tarification que si une péréquation suffisante existe entre les quartiers centraux et les quartiers périphériques. Cela suppose une volonté politique forte et durable.

\section{Dégager des moyens de financement efficaces}

La question de l'ampleur des sommes à mobiliser est souvent mise en avant comme la difficulté majeure. De notre point de vue, si on répond efficacement aux trois questions clefs et à la question centrale de la politique tarifaire, alors on trouve des financements. Répondre à ces questions constitue d'ailleurs souvent des « conditionnalités » justifiées de l'aide internationale, dont les sources sont disponibles, multiples et pas toujours complètement utilisées. Les recommandations du groupe de travail largement partagées par l'Agence française de développement (AFD) et la Banque mondiale sont les suivantes :

46 - si les risques de change sont probables, il faut éviter des solutions concessives et séparer l'exploitation du service (du type affermage ou régie) de la réalisation des investissements principaux, en maîtrise d'ouvrage publique et financement public. Il faut éviter, en effet, de faire emprunter des opérateurs privés en monnaie forte alors que les recettes sont libellées en monnaie locale;

47 - face à ce risque de change, la mobilisation de l'épargne locale souvent abondante et celle des expatriés qui cherchent à investir dans leur pays d'origine constituent une voie à privilégier, à condition de sécuriser les placements dans ces investissements à long terme, relativement sûrs, par des mécanismes de garantie ;

48 - il faut promouvoir des prêts directs aux autorités locales importantes ou, à défaut, faire prêter par une institution financière spécialisée dans les prêts aux autorités locales, qu'il faudrait inciter à créer si elle n'existe pas, cette institution bénéficiant elle-même des prêts des bailleurs de fond internationaux et étant en capacité de mutualiser les risques et de garantir les remboursements, sous l'égide de l'État ; 
49 - il faut enfin chercher à dégager des moyens budgétaires de l'État et des autorités locales à travers des mécanismes de taxe ou de fiscalité affectées à l'équipement urbain. La stabilisation de l'habitat spontané et le processus de développement économique qu'elle permet sont de nature à enclencher un cercle vertueux.

\section{Choisir le mode de gestion et l'opérateur sur des bases objectives}

\section{Sortir d'un débat trop idéologique}

50 L'attention est trop souvent portée sur le choix entre les solutions publiques ou privées. Voici le type de critiques généralement entendues, selon le point de vue adopté :

51 - aux régies ou aux solutions publiques en général, on reproche la mauvaise gestion, la qualité médiocre du service, le mauvais entretien du patrimoine (taux de fuites élevé), une motivation insuffisante des personnels, une attention insuffisante accordée aux attentes des usagers et un recouvrement insuffisant des factures. Les avantages reposent sur une maîtrise publique, en principe, plus complète du service et sur l'implication plus grande des responsables publics ;

52 - aux opérateurs privés, généralement internationaux, il est reproché de "faire de l'argent ou du profit » en exploitant un service public de base et de ne pas pouvoir, de ce fait, proposer des tarifications sociales pour les plus pauvres, de mettre en cause la maîtrise publique du service en raison de la dissymétrie du rapport de force ce qui peut conduire à une véritable perte de souveraineté. Les avantages reconnus reposent généralement sur l'efficacité, la réactivité, la bonne gestion des ressources humaines, l'utilisation de solutions techniques adaptées, la mutualisation des best practices.

53 Aux deux séries de solutions peuvent être souvent imputés des phénomènes de corruption de nature différente.

\section{Constater la pluralité des solutions existantes}

Du côté de la gestion publique, il y a la régie directe, mais aussi la régie autonome ou l'établissement public (national ou local). Des partenariats peuvent également être construits à prépondérance publique, de nature soit institutionnelle (société d'économie mixte) soit contractuelle avec des opérateurs apportant leur savoir faire sous forme de marché public de prestation de service ou d'assistance à l'exploitation. Des partenariats public-privé peuvent également être conçus dans lesquels le partenaire public joue un rôle d'autorité organisatrice et le partenaire privé est impliqué dans l'exploitation sous forme de « gestion partagée » (Tripoli au Liban) ou de gestion déléguée sous ses différentes formes (régie intéressée, affermage, licence ou concession). Il y a partage et transfert des risques de toutes natures, susceptibles d'être portés par le partenaire privé. Ces opérateurs privés peuvent être locaux, nationaux ou internationaux dans les opérations les plus importantes et complexes.

Des sociétés mixtes peuvent constituer une voie intéressante pour permettre l'émergence d'opérateurs privés nationaux en leur transférant des savoir-faire. 
Des acteurs du type association, communautés ou ONG peuvent également être chargés de gérer le service, notamment dans des zones rurales ou dans des quartiers urbains périphériques.

Si les organisations internationales ne sont pas elles-mêmes porteuses de solutions $a$ priori et quelquefois discutables, elles peuvent jouer un rôle utile dans les diagnostics préalables au choix. Ce « libre choix » doit pouvoir être remis en cause (« réversibilité » du choix) sans difficulté excessive, notamment à l'échéance d'un contrat. Des solutions progressives peuvent être retenues, les responsabilités confiées aux opérateurs privés s'accroissant au fur et à mesure que la confiance se construit et que les apports respectifs se précisent.

Une nouvelle politique de l'eau et de l'assainissement pour les grandes villes algériennes

Après des allers et retours périodiques entre des solutions centralisées publiques (ADE et ONA) et des solutions plus décentralisées, l'Algérie s'est engagée récemment, dans le cadre de son nouveau Code de l'eau, dans une politique cherchant à associer des opérateurs internationaux à l'exploitation des services des grandes villes (Alger puis Constantine, Oran et Annaba) à travers des contrats impliquant le partenaire privé dans la gestion (contrat d'Alger), avec une perspective ultérieure possible de gestion déléguée (affermage).

\section{Privilégier des relations contractuelles assorties de mécanismes incitatifs (bonus-malus), y compris en cas de gestion publique}

Le contrat est une voie privilégiée pour expliciter les objectifs, mettre au point un cahier des charges et des outils de suivi, de contrôle et d'évaluation, assortis de sanctions positives ou négatives. C'est un moyen d'inciter les parties au contrat à aller dans la bonne direction et à coopérer et de dissocier clairement les responsabilités entre l'opérateur et l'autorité publique.

Force est de constater qu'il est généralement plus facile d'engager une démarche contractuelle avec des opérateurs privés que de faire évoluer simultanément le management d'un opérateur public et la prise de responsabilité par l'autorité organisatrice. La solution privée suppose que l'autorité publique s'est mise, au préalable, en situation d'assumer son rôle, de contrôler l'opérateur et de garder le cap, en général avec une assistance extérieure.

\section{Éviter de faire porter à l'opérateur les défaillances de l'autorité publique}

60 Les échecs des contrats privés résultent souvent des défaillances des autorités publiques qui répugnent à assumer leur rôle (politique tarifaire et définition des grandes finalités en particulier) ou ne respectent pas leurs engagements contractuels. Il en va d'ailleurs de même en cas de gestion publique par confusion des rôles et manque de courage politique vis-à-vis des salariés et des usagers. Dans tous les cas, la corruption, qu'elle soit diffuse (clientélisme, népotisme) ou qu'elle constitue une condition ou une incitation à l'attribution du contrat, a des effets très négatifs. 


\section{Conclusion}

61 Comment mettre en mouvement les acteurs publics, faire en sorte qu'ils s'approprient les objectifs à atteindre, qu'ils prennent les dispositions voulues pour s'engager dans des actions de progrès et des processus pluriannuels de résolution des problèmes?

Les calendriers doivent être réalistes. Il vaut mieux des premières réponses limitées mais pour tous plutôt que des solutions trop ambitieuses, étalées dans des calendriers trop longs et forcément inégalitaires. Si elles trouvent des solutions convenables, les trois questions clefs et la politique tarifaire sont évidemment de nature à faciliter l'atteinte rapide de résultats tangibles. Il faut donc un " déclic » initial conduisant les pouvoirs publics à s'attaquer sérieusement au problème, en en faisant une réelle priorité de l'Agenda politique.

Des mécanismes de suivi et d'émulation entre villes et pays sont nécessaires : un forum s'appuyant sur des dispositifs de la convention de Barcelone devrait être en mesure d'assurer le suivi des résultats et les comparaisons, d'organiser des échanges d'expérience pour diffuser les bonnes pratiques, en s'appuyant sur les organismes existants (Plan d'action pour la Méditerranée, Institut méditerranéen de l'eau et Banque mondiale) avec le soutien de l'Union européenne. C'est en tous les cas un moyen indispensable pour maintenir la pression et susciter l'émulation entre les acteurs qui peuvent ainsi se conforter mutuellement.

Des procédures d'auto-évaluation (diagnostic de l'état des problèmes) ou d'évaluation mutuelle ou par tierce partie pourraient également être préconisées pour favoriser les prises de consciences et convaincre les autorités publiques ( «nul n'est prophète en son pays »!) de s'engager dans des processus de changement. Un guide d'auto-évaluation a été élaboré à cette fin par le groupe de travail.

Sur la base d'une doctrine et de recommandations désormais relativement claires, il paraît possible d'aller de l'avant. Des avancées récentes dans certains pays (Algérie, Maroc) montrent que le moment paraît venu de prendre ces problèmes à bras-le-corps, pour enfin commencer à atteindre les objectifs fixés, il y a déjà trop longtemps !

\section{BIBLIOGRAPHIE}

Agence Française de Développement, 2006, Rapport annuel, Paris.

Benoit G., Comeau A., 2005, Méditerranée : les perspectives du Plan Bleu sur l'environnement et le développement, éditions de l'Aube et Plan Bleu.

Brook P. J., Smith S. M., 2001, Contracting for Public Srvices: Output-based Aid and its Applications, The World Bank Group.

Conseil Mondial de l'Eau, 2003, Financer l'eau pour tous, Rapport du panel mondial sur le financement des infrastructures de l'eau présidé par Michel Camdessus. 
Conseil Mondial de l'Eau, 2006, Report of the Task Force on Financing Water for All, Rapport de Paul Van Hofwegen sous la présidence d'Angel Gurria.

Institut de la Gestion Déléguée, 2004, Indicateurs de performance « Eau potable et Assainissement »Charte des Services Publics Locaux.

Institut de la Gestion Déléguée, 2006, Gouvernance, partenariat et financement pour les services essentiels, Comité pour la Charte des services essentiels, Rapport du groupe de travail.

Institut de Prospective Économique du Monde Méditerranéen, 2007, Eau et Assainissement dans les villes et pays riverains de la Méditerranée, Rapport du groupe de travail sous la présidence de Claude Martinand.

Partenariat mondial pour l'aide basée sur les résultats, 2005, L'aide basée sur les résultats, OBApproaches, note $\mathrm{n}^{\circ} 5$, mars 2005.

PNUE, PAM et Plan Bleu, 2006, «Faire face aux crises et pénuries d'eau en Méditerranée », Les Notes du Plan Bleu, $\mathrm{n}^{\circ} 4$.

Tourret J.-C., 2005, Les villes méditerranéennes dix ans après Barcelone, Mairie de Barcelone.

UNDP, 2006, Au-delà de la pénurie : Pouvoir, pauvreté et crise mondial de l'eau, Rapport mondial sur le développement humain.

Plan des Nations unies pour le développement, Agence européenne de l'environnement, 1999, State and Pressures of the Marine and Coastal Mediterranean Environment.

\section{RÉSUMÉS}

Notre mer commune est quasi fermée et ce vase clos reçoit et concentre l'ensemble des pollutions émises par les villes et pays riverains, ainsi que par les activités maritimes ellesmêmes. De multiples conventions, plans, programmes et fonds ont défini et tenté de mettre en œuvre une réponse à ce problème depuis la convention de Barcelone de 1975 et le Plan d'action pour la Méditerranée (PAM). L'article s'interroge sur les raisons qui ont conduit à des résultats mitigés, certes variables selon les pays, mais insuffisants sur le terrain. Faisant la synthèse d'études de cas retraçant des réussites et les difficultés rencontrées, il fait des recommandations susceptibles de conduire plus rapidement à des résultats tangibles, en insistant sur la qualité de la gouvernance et la gestion de la demande, et en signalant que seule une prise de conscience au plus haut niveau, celui des chefs de gouvernement et non pas des ministres de l'environnement, pourra déclencher le passage à l'action. Il fait des propositions pour développer l'échange d'expériences entre les deux rives de la Méditerranée. Ces éléments sont de nature à faire émerger une politique commune pour gérer ce bien public transnational qu'est la qualité de l'eau en Méditerranée.

The Mediterranean is almost locked. It concentrates various types of pollutions rejected by surrounding urban areas and countries and by the activites related to the sea. Nummerous conventions, plans, programmes and funds have defined and tended to implement a relevant response to this problem, since the Barcelona convention (1975) and the Action Plan for the Mediterranean. This paper addresses the reasons why all these documents have had only uncertain if not poor results. The results vary a lot in various countries but they are quite unsufficiant in the field. The authors propose a synthesis of several case-studies. They underline the difficulties faced by the actors involved in various projects. They also put the stresss on some success stories. They make recommendations in order to get tangible results more rapidly, putting the stress on the quality of the governance and on the capacity to keep the consumption 
under control. The implementation of efficient policies in this field will come only if the highest level, that is the heads of states or governements and not only the minsiters of environnement, become more aware of this important issue. Besides, the authors also recommend the development of exchanges of experiences between both banks of the Mediterranean Sea. Finally, they wonder if the surrounding countries should not carry out a common policy in order to manage this common public good: the water in the Med region.

\section{INDEX}

Mots-clés : eau, pollution, activité maritime, environnement Index géographique : Méditerranée

\section{AUTEURS}

\section{CLAUDE MARTINAND}

Vice-président du Conseil général des Ponts et Chaussées, Président de l'Institut de la gestion déléguée.

MARIE-JOËLLE KODJOVI

Chargée de mission à l'Insitut de la gestion déléguée. 